This is the final peer-reviewed accepted manuscript of:

M. Magno, V. Jelicic, B. Srbinovski, V. Bilas, E. Popovici and L. Benini, "Design, Implementation, and Performance Evaluation of a Flexible Low-Latency Nanowatt Wake-Up Radio Receiver", in IEEE Transactions on Industrial Informatics, vol. 12, no. 2, pp. 633-644, April 2016, doi: 10.1109/TII.2016.2524982.

The final published version is available online at:

https://doi.org/10.1109/TII.2016.2524982

Rights / License:

The terms and conditions for the reuse of this version of the manuscript are specified in the publishing policy. For all terms of use and more information see the publisher's website.

This item was downloaded from IRIS Università di Bologna (https://cris.unibo.it/)

When citing, please refer to the published version. 


\title{
Design, Implementation, and Performance Evaluation of a Flexible Low-Latency Nanowatt Wake-Up Radio Receiver
}

\author{
Michele Magno, Member, IEEE, Vana Jelicic, Student Member, IEEE, \\ Bruno Srbinovski, Student Member, IEEE, Vedran Bilas, Senior Member, IEEE, \\ Emanuel Popovici, Senior Member, IEEE, and Luca Benini, Fellow, IEEE
}

\begin{abstract}
Wireless sensor networks (WSNs) have received significant attention in recent years and have found a wide range of applications, including structural and environmental monitoring, mobile health, home automation, Internet of Things, and others. As these systems are generally battery operated, major research efforts focus on reducing power consumption, especially for communication, as the radio transceiver is one of the most power-hungry components of a WSN. Moreover, with the advent of energy-neutral systems, the emphasis has shifted toward research in microwatt (or even nanowatt) communication protocols or systems. A significant number of wake-up radio receiver (WUR) architectures have been proposed to reduce the communication power of WSN nodes. In this work, we present an optimized ultra-low power (nanowatt) wake-up receiver for use in WSNs, designed with low-cost off-the-shelf components. The wake-up receiver achieves power consumption of $152 \mathrm{nW}$ (with $-32 \mathrm{dBm}$ sensitivity), sensitivity up to $-55 \mathrm{dBm}$ (with maximum power of $1,2 \mu \mathrm{W}$ ), latency from $8 \mu \mathrm{s}$, tunable frequency, and short commands communication. In addition, a low power solution, which includes addressing capability directly in the wake-up receiver, is proposed. Experimental results and simulations demonstrate low power consumption, functionality, and benefits of the design optimization compared with other solutions, as well as the benefits of addressing false positive (FP) outcomes reduction.
\end{abstract}

Index Terms-Nanowatt wake-up radio receiver (WUR), power optimization, ultra-low power, wireless sensor networks (WSNs).

Manuscript received August 04, 2014; revised October 09, 2015; accepted December 16, 2015. Date of publication February 05, 2016; date of current version March 29, 2016. This work was supported by the project Scientific co-operation between Eastern Europe and Switzerland (SCOPES) and SWISS NATIONAL FUNDATION (SNF) under Grant IZ74Z0_160481, Science Foundation Ireland (SFI) Marei Centre and Analog Devices at University College Cork (UCC). Paper no. TII-15-0772.

M. Magno and L. Benini are with the Department of Electrical, Electronic, and Information Engineering, University of Bologna, Bologna 40126, Italy, and also with the Swiss Federal Institute of Technology Zurich, Zurich 8092, Switzerland (e-mail: michele.magno@iis.ee.ethz.ch; luca.benini@unibo.it).

V. Jelicic and V. Bilas are with the Faculty of Electrical Engineering and Computing, University of Zagreb, Zagreb HR-10000, Croatia (e-mail: vana.jelicic@fer.hr; vedran.bilas@fer.hr).

B. Srbinovski and E. Popovici are with the Department of Electrical and Electronics Engineering, University College Cork, Cork, Ireland (e-mail: e.popovici@ucc.ie).

\section{INTRODUCTION}

W IRELESS sensor networks (WSNs) have been recognized as an enabling technology for a large variety of applications, including smart homes and cities, agriculture, transportation, health and fitness, entertainment, and structural health monitoring [1]. Strict energy constraints of batterypowered wireless sensor nodes have introduced the necessity of energy awareness in both software and hardware solutions. Reducing the communication power consumption of WSN nodes is important, as the radio transceiver is one of the components with the highest power consumption. Optimizing the power consumption of the wireless transceiver can free up power budget to add much more functionality. In addition, since the battery size is decisive in determining the size of batteryoperated systems, low-power circuits could enable smaller batteries and lead to miniaturization required by many applications such as wearable WSNs, medical body area networks and implantable devices.

To establish communication, two radios (the receiver and the transmitter) need to be synchronized as a message can be received only if the radio is in its listening state, and idle listening consumes significant power. Hence, a significant design effort is required to alleviate this power waste. To reduce communication power consumption, several techniques have been proposed [2]-[7] for lowering or eliminating the power wasted due to idle listening of the transceiver. Duty cycling is a common technique to reduce the idle mode energy consumption which consists of switching from listening mode to sleep mode [2]. However, while duty cycling helps save power, it can severely limit the network reactivity as the radios are OFF (or in the sleep state) and they cannot receive messages. Duty cycling is in general a synchronous technique, where the radio is woken up for a fixed or adaptive time interval to listen if there is any relevant incoming message. However, there are three types of communication techniques: synchronous, pseudo-asynchronous, or pure asynchronous [3].

From a power consumption perspective, asynchronous schemes are considered by far the most efficient, and the most effective realization of an asynchronous communication which is achieved by reducing or eliminating idle listening using a wake-up radio receiver (WUR) [7]. Such a device is coupled with the main radio transceiver having the role of listening 
continuously to the transmission medium and waking up the main transceiver upon detection of an incoming message.

There are numerous features that a wake-up radio device must support to become effective. First of all, the power consumption of the WUR has to be orders of magnitude lower than that of the main transceiver in the receiving mode. Other important features are greater sensitivity, robustness to interference, selectivity, and low latency. Typically, sensitivity (the weakest signal the receiver is able to sense) is the most important optimization goal with the ultimate aim being to fix the sensitivity of the main transceiver at a much lower power. The sensitivity is directly related to the communication range: the greater the sensitivity, the longer the range. However, improving the receiver sensitivity is typically constrained with increased power consumption.

In this paper, an optimized architecture for an ultra-low power, low-cost wake-up receiver is presented, considering all the constraints and specifications described above. In more detail, the contributions of this paper are as follows.

1) A set of optimization techniques targeting ultra-low power wake-up receivers.

2) The design and implementation of a wake-up receiver which has only one ultra-low power comparator as an active component, and an optional ultra-low power microcontroller for addressing capability. The wake-up receiver is built using a minimal number of low-cost, off-the-shelf components.

3) Experimental validation of the proposed approach, in terms of power consumption, sensitivity, range, data rate, functionality, and addressing capability when connected to a microcontroller.

4) Comparison with existing wake-up receivers and a media access control (MAC) protocols (with and without addressing) to evaluate the benefits of the proposed solution.

This paper is organized as follows. Section II reviews the related work. In Section III, the architectural considerations are discussed, while Section IV presents the submicrowatt wakeup receiver architecture proposed in this work. Finally, Sections $\mathrm{V}$ and VI present experimental setup and experimental results, respectively, with three different implementations. Section VII concludes this paper.

\section{RELATED WORK}

Research on wake-up radio use in WSN to reduce power consumption has been prolific in recent years. With a variety of proposed methods and techniques, three groups of radio frequency (RF) wake-up systems can be identified: fully passive circuits, semi-active circuits, and fully active circuits.

\section{A. Fully Passive Wake-Up Circuits}

Wake-up systems with fully passive circuits work without any power supply as the circuit harvests energy from the radio communication and uses it to generate an interrupt. They are mostly realized using charge pump, Schottky diodes, and CMOS or MOSFET technology. However, the fully passive receivers only detect activity in the communication channel and cannot distinguish a wake-up signal from other RF activity. Moreover, it is impossible to receive commands or data to address a node. Another limit of these circuits is a short communication range compared to most WSN applications. Usually, they have a sensitivity around $-25 \mathrm{dBm}$ which implies the use of higher transmission power or (where possible) a bigger antenna to extend the range to tens of meters.

Due to these limitations, they are more suitable for short-range applications, which do not need any addressing mechanism. For example, they can be used in some implantable chips, body area networks, near field communication, and Radio-Frequency identification (RFIC). Despite that, some of the interesting zero-power building blocks are present in a number of passive WUR architectures, such as passive rectifiers with interrupt block, with many designs being presented in the literature in recent years [5]. Although the zero-power consumption feature is very appealing, we do not implement, it in our proposed solution, as we are focused on solutions for a long range with sensitivity from -30 to $-55 \mathrm{dBm}$ that would give benefit to a wider range of WSN applications. For this reason, the proposed approach uses a semi-active receiver with a single-stage rectifier instead of a multistage charge pump used in the RFID solutions, as the main goal is maximizing the communication range. In fact, passive RFID systems are achieving only a few centimeters of range, while our solution can achieve several meters.

\section{B. Semi-Active Wake-Up Circuits}

The majority of the proposed design approaches are semiactive, whereas the minority of the receiver's components are battery powered. The most common approach is based on an envelope detector, implemented with passive components (Schottky diodes, MOSFETs, or ad-hoc Integrated Circuits (ICs) for the radio front end) followed by an active component (a comparator) to generate an interrupt. The research in [8][10] presents such architectures for ultra-low power WURs for WSN devices achieving a reduction of sensor node listening activities and drastically reducing the overall network power consumption. The solutions use a multistage rectifier rather than a single-stage without elaborating this choice or methods for power and sensitivity measurement.

We use a single-stage rectifier combined with an ultra-low offset comparator. In our solution, this is a significant design difference with respect to other solutions as a combination of a single-stage rectifier and a comparator with an optimized offset voltage is able to achieve the minimal sensitivity possible with a passive front end $(-55 \mathrm{dBm})$. Moreover, we optimized the reference voltage source of the comparator eliminating the high delay due to long preamble needed in other solutions [8]-[10].

In [11], a very interesting solution with a $98-n W$ power consumption is presented. This solution is similar to the solutions presented above (using a rectifier and a comparator), but the custom CMOS rectifier was designed to achieve sensitivity of $-41 \mathrm{dBm}$. This sensitivity is lower (allowing a shorter range) than the one achieved in our approach. Also, we present an offthe-shelf solution which can reduce cost and implementation 
time. A previous work published in [12] a similar architecture. In this work, more details of the different design parameters are presented, and a more complete set of evaluations with simulation and in-field measurements has been performed. Finally, the benefits of the addressing capability have been shown in the experimental results.

\section{Fully Active Wake-Up Circuits}

This group of circuits use active components both for the rectifier and the interrupt generator. In [13], a solution with a rectifier, high-band baseband amplifier, and the wake-up signal recognition is presented. The sensitivity of $-47.2 \mathrm{dBm}$ is good, similar to our obtained results. However, as for all fully active solutions, the power consumption of more than $6 \mu \mathrm{W}$ is much higher than for our solution. The aim of this paper is to design a high sensitivity and under $\mu \mathrm{W}$ solution. The authors in [15] propose an architecture featuring a low-noise amplifier and fully active solution to achieve sensitivity of $-89 \mathrm{dBm}$. However, the solution suffers from very high power consumption of few $\mathrm{mW}$, which offsets the main benefits of the wake-up receiver. In [16], CMOS chip including envelope detector, low-noise baseband amplifier, mixed-signal correlation unit, and auxiliaries for stand-alone operation is presented. The achieved performance is very interesting: $-71 \mathrm{dBm}$ of sensitivity at $868 \mathrm{MHz}$ and $2.4-\mu \mathrm{W}$ power consumption (at $1 \mathrm{~V}$ ) with a quite high latency of $7 \mathrm{~ms}$. In this work, we present an off-the-shelf solution which can be prepared without large nonrecurring engineering investments and ramp up to production volume of new dedicated chips. Moreover, our solution achieves better performance in power consumption, latency, and flexibility thanks to the frequency independency.

\section{Media Access Control With Wake Up Radio}

A thorough survey of various wake-up schemes and their advantages over the wake-on (duty-cycling) schemes is presented in [3]. It is shown that the wake-up radio without addressing presented in [9] has an advantage over the other schemes due to its very low power consumption and low latency. However, an addressing mechanism is needed to reduce the power consumption used during network formation, and if the wake-up receiver can receive some command, the MAC data communication protocol can be simplified for low power consumption. There are two ways of implementing the addressing mechanism: 1) custom circuit for addressing/address comparison [14]; and 2) using a generic microcontroller [10]. The addressing methodology will have implications on the overall power budget and will influence the selectivity of the system. This confirms the importance of the capability of receiving data, which is included in our approach.

The approach proposed in this paper significantly extends the state-of-the-art with respect to power consumption, latency, and sensitivity. Moreover, it gives guidelines and insights to take into account when designing a WUR with off-the-shelf components. The selection of the single-stage rectifier is justified, and its advantage over the multistage architecture is demonstrated with measurements. The importance of the offset voltage of the

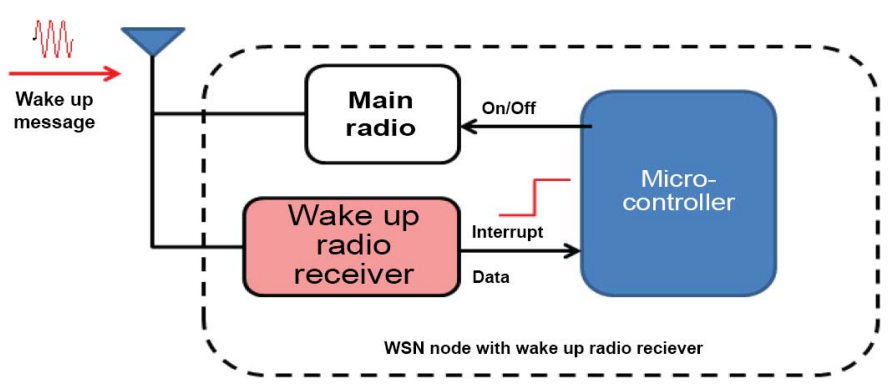

Fig. 1. Generic block diagram of a wireless node with a separate WUR.

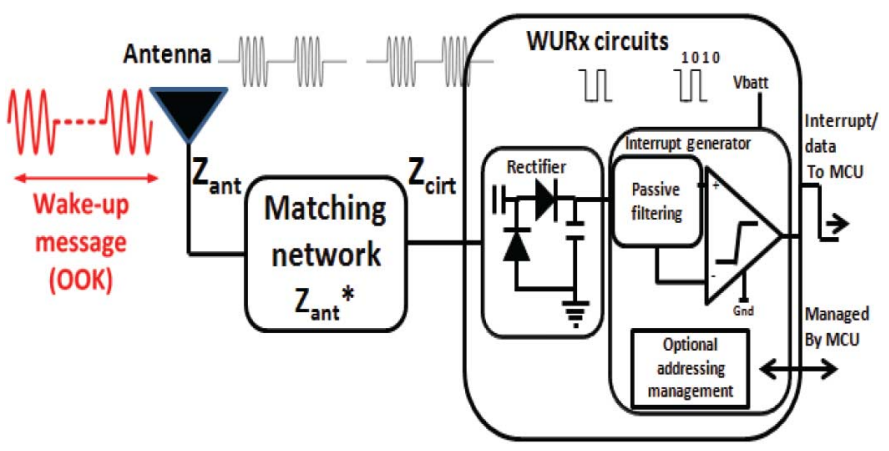

Fig. 2. Block diagram of the wake-up receiver with the impedance matching of WUR and the antenna to maximize the power transferred.

comparator is also described in detail together with the optimization of the reference voltage source generator. To the best of the authors' knowledge, this is the first paper which takes into account all aspects of wake-up receiver design, from the matching network, rectifier, stages of rectifier, interrupt generator, and from false wake-ups to the addressing capabilities.

\section{WUR DESIGN BLOCKS}

In this section, we present the WUR blocks and the design consideration. Fig. 1 shows a generic block diagram of a wireless node with a WUR. The design of the wake-up radio requires careful consideration of design issues in $\mathrm{RF}$, analog electronics, and digital and system design to carefully evaluate the following tradeoffs:

1) wake-up range versus energy consumption;

2) wake-up range versus delay;

3) same-band versus different-band wake-up radio;

4) addressing versus not addressing.

Fig. 2 shows the main blocks required to design a WUR. The wake-up message is received by the WSN node trough the antenna. Then, a matching network is needed to provide maximal power transfer between the antenna (typically with $50 \Omega$ impedance) and the rest of the circuit. The rectifier converts the input radio signal into a dc signal. In general, in order to keep the power consumption as low as possible, the rectifier is passive. Once the dc signal is generated, it needs to be "processed" by the interrupt generator to alert further logic, or the microcontroller. The interrupt generator also consists of a passive/active filtering or addressing circuitry to activate the interrupt according to the address. In the following sections, all the blocks are analyzed in detail. 


\section{A. Frequency, Sensitivity, and Antenna}

The selection of an operating frequency band for the wakeup receiver is crucial since it will affect the size of the receiving antenna, the operating range of the system and the matching network selection, and availability of the required passive components. To get an idea how the frequency affects these features of the wake-up receiver, the Friis equation is used

$$
P_{r}=\frac{P_{t} \cdot G_{r} \cdot G_{t} \cdot \lambda^{2}}{(4 \pi)^{2} \cdot d^{n}}
$$

$P_{r}$ is the power received from an antenna, $P_{t}$ is the transmission power, $G_{r}$ and $G_{t}$ are the gains of the receiving and transmitting antenna, respectively, $d$ is the transmission distance, $\lambda$ is the wavelength of the frequency used, and $n$ is the path loss exponent. $P_{r}$ is usually expressed in $\mathrm{dBm}$ and the sensitivity of the receiving circuits equals the lowest value of $P_{r}$ at which the circuit can receive the wake-up message reliably. Thus, from (1), considering $P_{t}=+10 \mathrm{dBm}, G_{r}$ and $G_{t}=1 \mathrm{dBi}$ and frequency of $868 \mathrm{MHz}$, the wake-up circuit with sensitivity $-50 \mathrm{dBm}$ can achieve the maximal theoretical range of $34 \mathrm{~m}$. Accordingly, lower frequencies allow longer ranges.

However, decreasing the frequency increases the antenna length. Depending on the application scenario, it may be impossible to use a large size antenna (i.e., for body area networks) so migration to higher frequency has to be considered. If lower frequencies are part of the system specification, then one has to consider that antenna miniaturization at these frequencies would induce significant losses which are reflected in reduced communication range or higher transmission power. Moreover, the application scenarios often force usage of the same antenna/frequency as for the radio used in the network. In fact, in many applications, to achieve low cost and small form factor, the wake-up signal has to be generated from the main radio. The most commonly used frequencies in WSNs are $868 \mathrm{MHz}$ and $2.4 \mathrm{GHz}$ which are the most popular for the wake-up communications as well, although examples using other frequencies can be found in [5]-[9].

\section{B. Modulation}

The WUR is a simple radio receiver with ultra-low power consumption which calls for a very simple architecture. This design goal also imposes constraints on the modulation used for the wake-up radio. If the modulation used is highly complex [e.g., phase shift keying (PSK), quadrature phase shift key (QPSK), and others] the wake-up receiver demodulation circuits will be more complex and will require more power. The most popular modulation scheme used in wake-up receivers is the on-off keying (OOK) [3]. OOK denotes the simplest form of amplitude-shift keying (ASK) modulation that represents digital data with the presence or absence of a carrier wave. This modulation works as a Morse code, where, to transmit a digital signal (1 and 0), a carrier wave is switched ON and OFF, respectively. This simple modulation allows a drastic simplification of the WUR circuitry and provides power reduction opportunities as will be presented in the following sections. In the rest of this paper, we assume that OOK modulation is used.

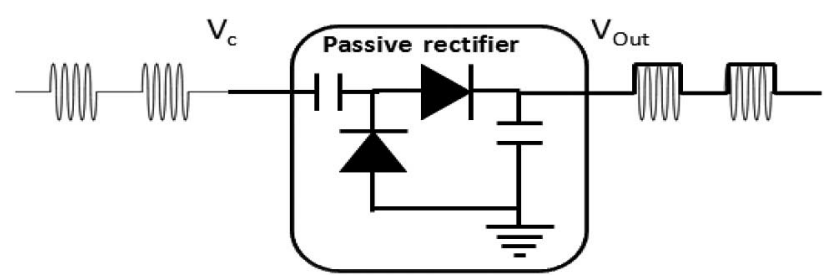

Fig. 3. Envelope detector architecture with two diodes. The input of the envelope is the RF signal while the output is the envelope of the signal in the dc domain.

\section{Matching Network}

As mentioned earlier, the maximum power of the received radio signal available on the antenna (1) is transferred to the rest of the circuit when the impedance of the receiver circuit $\left(Z_{\text {cirt. }}\right)$ equals the conjugate of the antenna impedance $\left(Z_{\text {ant }}\right)$. Since, in general, $Z_{\text {cirt }}$ is not equal to $Z_{\text {ant }} *$, a matching network circuit is required in between to achieve this important goal and not to waste precious received power.

The effect of mismatches is highly visible at frequencies over $300 \mathrm{MHz}$ when significant amount of the RF power can be reflected when it reaches an RF circuit. The most common measure of mismatch is return loss expressed in $\mathrm{dB}$ or by $\mathrm{S}$ parameters. If an RF signal is incident on the input side of the circuit, some of the signal is reflected and some is transmitted through the circuit. The ratio of the reflected electromagnetic field to the incident field is the reflection coefficient (S11).

The main goal of impedance matching is the reduction or elimination of the reflected RF signal, which involves measuring the reflected power and designing the matching network. It is important to note that the matching network is strictly linked with the selected working frequency. Thus, although a receiver could work in different frequency domains, the matching network limits this freedom.

\section{Rectifier}

To recover the information content from the received modulated carrier waveform, a rectifier is needed. As mentioned before, using OOK modulation enables building simple and passive demodulation circuit. The circuit function is to remove the residual carrier signal while preserving the modulating (envelope) waveform. A simple form of rectifier consists of a diodes detector connected to a capacitor from the output of the circuit to the ground (Fig. 3) which works as an envelope detector. The recovered envelope signal has lower amplitude and the same form as the original signal. The peak of the incoming ac signal must be higher than the bias voltage of the diode to allow the diodes to rectify the signal. Since the envelope detector circuit is basically a half-wave rectifier, there will be a dc level developed across capacitors.

One of the crucial requirements for WUR receiver circuit is to operate with weak input RF signals to achieve long range. For a typical WSN application operating at $2.4 \mathrm{GHz}$, at distances of 4-5 $\mathrm{m}$ between nodes, the RF signal power received on the antenna is already $-30 \mathrm{dBm}$ or less. As the peak voltage of the ac signal obtained at the antenna is very low, diodes with the lowest possible bias voltage are preferable. To have a 
high sensitivity WUR, zero-bias diodes should be implemented. Rectifiers implemented with a custom IC solution are present in the literature [11], but these are not commercially available and are out of the goals of this paper. In this work, we will use an envelope detector as a rectifier using commercial-of-the-shelf zero-bias diodes able to achieve sensitivity of $-56 \mathrm{dBm}$.

Rectifier's number of stages: For the ideal electronic components, the output voltage of the envelope detector $V_{\text {out }}$ rises with the number of the stages $N$

$$
V_{\text {out }}=2 N\left(V_{c}-V_{d}\right)
$$

where $V_{d}$ is the bias voltage of the diodes and $V_{C}$ is the voltage at the input of the envelope detector. Equation (2) confirms the requirement of zero-bias diodes. In reality, one could not continue adding as many stages as required to convert the input RF signal into a desired output voltage $V_{\text {out }}$, because (2) is an approximate equation which does not consider the effect of power wasted by adding diodes, capacitors, and Printed Circuits Board (PCB) lines as explained in [17]. Thus, increasing the number of stages $N$ reduces the whole efficiency of the envelope detector. This is particularly important for the wake-up receiver where the RF signal is very weak and $V_{d}$ becomes comparable with $V_{C}$.

Another important effect to be considered is that $V_{C}$ depends on the number of stages as well. From (3) and (4), as more stages are added, equivalent input resistance of the envelope detector $\left(R_{\mathrm{EQ}}\right)$ becomes smaller, the conductance $\left(X_{\mathrm{EQ}}\right)$ becomes higher and $V_{C}$ decreases

$$
\begin{aligned}
P_{r} & =V_{C} I=\frac{V_{C}^{2}}{R_{\mathrm{EQ}}} \quad V_{C}=\sqrt{P_{r}} R_{\mathrm{EQ}} \\
R_{\mathrm{EQ}} & \propto \frac{1}{N} ; \quad X_{\mathrm{EQ}} \propto N .
\end{aligned}
$$

Fig. 4 depicts the input voltage of the envelope detector $V_{C}$ relation to the equivalent resistance $R_{\mathrm{EQ}}$ for a constant received power $P_{r}=-30 \mathrm{dBm}$. Fig. 4 shows that $V_{C}$ increases with an increase of the $R_{\mathrm{EQ}}$, according to (3), and the measured value of $R_{\mathrm{EQ}}$ for different stages. Therefore, in order to maximize the range of the WUR receiver, we need to maximize the $V_{C}$ that is detected by the comparator, which depends also from the minimal offset voltage of the comparator self (see next section). So, it is very crucial to utilize a single-stage envelope detector. This is also the main difference between our architecture and previous works (i.e., [9]) where a double-stage rectifier has been used without any justification and resulting in reduced sensitivity.

\section{E. Interrupt Generator}

The function of this block is to generate an interrupt to a microcontroller with the shortest latency and minimal influences of interferences. This is the first block of the wake-up receiver operating with a dc signal and the enveloped data (Fig. 2). There is also an option to add the addressing capa-bility to this block to selectively wake up at the specific address and to generate digital data for the microcontroller or an IC. Despite the case of the RF energy harvesting [18], where multi-stage envelope detectors can provide a voltage higher than $1 \mathrm{~V}$

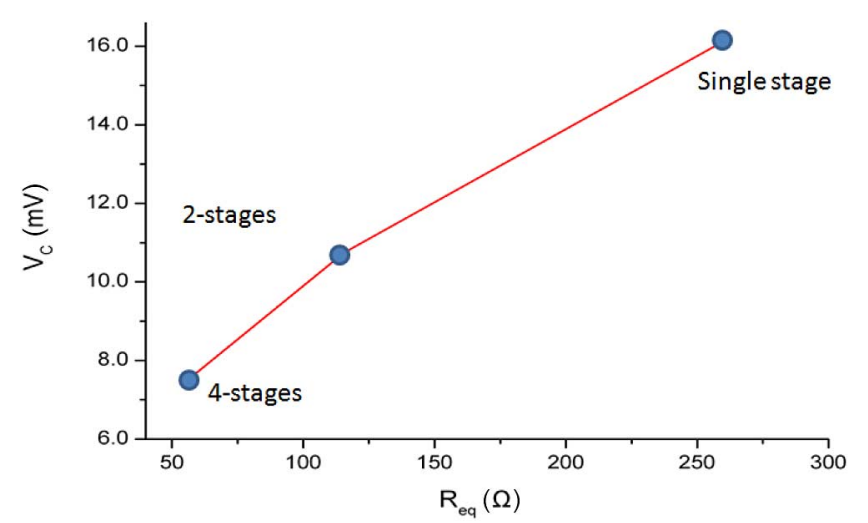

Fig. 4. $V_{C}$ as function of the equivalent resistance $R_{\mathrm{EQ}}$ for constant $P_{r_{i}}=-30 \mathrm{dBm}$. Increasing $R_{\mathrm{EQ}}$ by decreasing the numbers of stages $N$ will increase $\mathrm{V} c$.

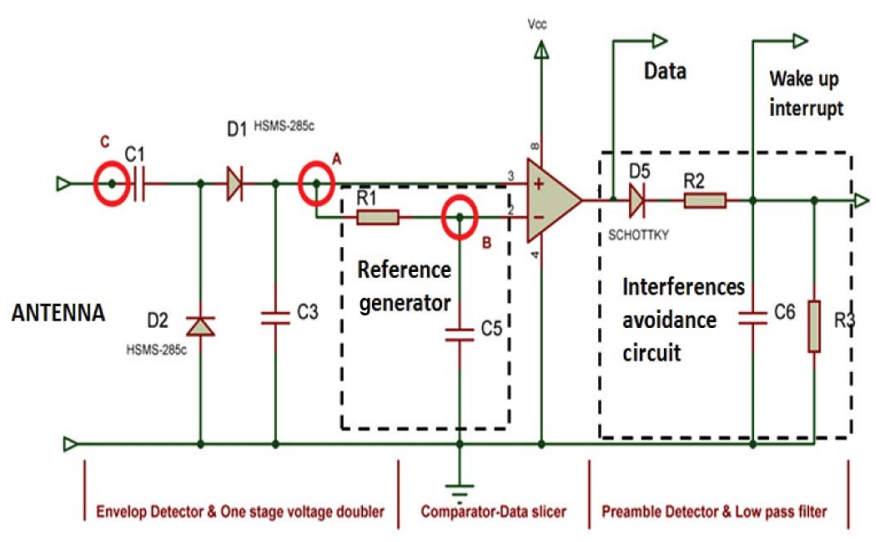

Fig. 5. Nanopower high-sensitivity WUR architecture.

and trigger an IC directly, as presented in previous sections, this is unfeasible for the weak RF signals typical for the WUR receivers. In fact, as Fig. 4 shows, with a received signal of $-30 \mathrm{dBm}$ or lower, the input voltage of the rectifier is only a few $\mathrm{mV}$ and adding stages to the envelope detector will decrease the input voltage wasting the multiplier effect. For this reason, this block needs to be supplied with battery as it generates an interrupt with higher voltage (at least $1.5 \mathrm{~V}$ ) able to wake up a microcontroller or an IC. The simplest and low power way to do this (the base to the most WURs) is using an ultra-low power comparator [8]-[10]. From the signal generated by the envelope detector, it is necessary to create both the reference voltage and the signal at the input of the comparator. If the low power consumption is the main constraint, this reference source can be passive using a simple Resistor-Capacitor (RC) low-pass filter. In the same way, it is possible to decouple the comparator input, filtering the output of the comparator with another RC filter. Fig. 5 depicts a wake-up radio architecture where a semi-passive interrupt generator with the comparator as the only active component is present. This architecture is simple, consumes negligible power, and although it is not possible to perform addresses parsing, the data are preserved for further blocks with addressing capability.

Fig. 6 shows the output voltage of an interrupt generator built with a comparator compared to the RF signal arriving to the antenna. The interrupt generator gives a digital envelope of the 


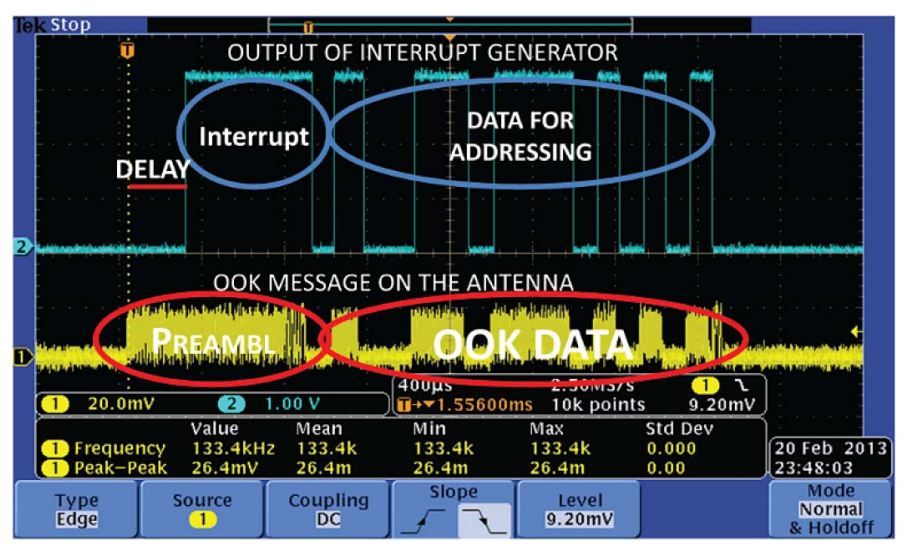

Fig. 6. OOK signal on the antenna of the WUR (plot 1 down /yellow) and data out from the interrupt generator (plot 2 up/blue).

original OOK message at the output. This feature is very interesting for developing further integrated or discrete circuits, to automatically detect the addressing or use the microcontroller, which is woken up through the interrupt generator to process the data and make the decision to wake up the radio or else go back to sleep.

Due to low-pass filtering at the comparator output, the interrupt is generated only if the preamble (Fig. 6) is longer than $400 \mu \mathrm{s}$ (that can be tuneable by the Resistor-Capacitor-Resistor (RCR) filter). This will protect the circuits from interference but will create a delay shown in Fig. 6. This is another tradeoff of the wake-up receiver which requires careful design. The dura-tion of the delay can be set by changing the value of $R_{2}, C_{6}$, and $R_{3}$ in Fig. 5 .

The main criteria for selecting the comparator circuit are: 1) power consumption; 2) input offset voltage $V_{\mathrm{IO}}$; and 3 ) propagation delay.

Bearing in mind that the goal is to build a wake-up receiver circuit that will have very low power consumption, the quiescent current consumption of the comparator must be in the order of few nano-Amps.

The input offset voltage is another important parameter of the comparator. It is defined as the differential input voltage to apply in order for the comparator to be at a toggling level [19]. As further discussed in [19], the input offset voltage also limits the resolution of a comparator. Therefore, for very small signals (in the same order as the input offset voltage $-V_{\mathrm{IO}}$ ), the comparator toggles at an undesired rate or does not toggle at all. To increase the sensitivity of the wake-up receiver and avoid the possible issues discussed above, the input offset voltage of the comparator has to be lower than the minimal expected output voltage of the envelope detector circuit, $V_{C}$.

\section{F. Addressing Management Circuit}

The addressing management circuit is an optional subsystem in charge to decode the data received by WUR and generate a further trigger only when the address is recognized. If it is present, this is the first digital part which is woken up from the interrupt generation. The addressing circuit provides a digital interface for the main microcontroller to be set with the desirable address. When the addressing circuit is not present, the main microcontroller can process the data and evaluate its address. There are different implementations possible for the addressing generator; however, the majority uses an ad-hoc designed ASIC or an ultra-low power microcontroller. It is important to notice when an addressing circuit is present, the final wake up signal is generated only after the address is received. This increases the overall wake up latency but can reduce the number of false positives (FPs) and, in turn, the power consumption at the system level. The wake-up time with addressing is proportional to the length of the address and baud rate with Wakeup Time $=N_{\text {bits }} * T_{\text {bit }}$ where $\mathrm{T}_{\text {bit }}=1 /$ Baud rate, and $N_{\text {bits }}$ is the number of the bits used for the address.

\section{Proposed Ultra-Low Power WUR}

This section presents the proposed design of a nanowatt WUR which uses only a comparator as an active component to evaluate and demonstrate the proposed analysis in a real development. Moreover, this section will present a method to use the WUR by adding addressing capability, keeping the power low. All the blocks presented in previous sections are described in detail.

The proposed architecture's (Fig. 5) comparator is the only component powered at all times. The main goals when designing this receiver are as follows:

1) ultra-low power, around or less than $1 \mu \mathrm{W}$;

2) high sensitivity, with at least $-35 \mathrm{dBm}$;

3) flexibility adaptable to different frequencies in ISM band with OOK modulation;

4) reactivity less than $100 \mu$ s for the first interrupt;

5) preservation of the received data, for further processing to perform addressing or receive commands;

6) optional ultra-low power and low-cost addressing management.

\section{A. Rectifier}

We built a single-stage rectifier with HSMS-285C diodes [22]. HSMS-285C is used in the proposed solution, as the requirements are to cover frequencies below $1 \mathrm{GHz}$; however, the same schematic we are presenting can be used for frequencies above $1.5 \mathrm{GHz}$ (HSMS 282x diodes). These diodes are optimized for incoming power lower than $-20 \mathrm{dBm}$ and offer sensitivity of $-57 \mathrm{dBm}$ that makes them the best solution on the market at the time this paper is written. The diodes are successfully used in several RF energy harvesting and wakeup applications [18], which confirm their performance in weak RF signal environments.

\section{B. Comparator}

Three different comparators were used in the presented study in order to evaluate the tradeoff of power and sensitivity. We selected TLV3691 from Texas instruments (TI) with ultra-low current consumption and 2.5-mV input offset, AS1976 from Austria Microsystems with 1.8-mV input offset and LPV7215 from TI with $0.5-\mathrm{mV}$ input offset. Table I shows the performance of the comparators in terms of power, sensitivity, and range. The input offset voltage of each selected comparator was measured using the method presented in [19]. The results in the 
TABLE I

WAKE-UP RAdio PERFormance For DifFERENT COMPARATORS AT 2 V SUPPLY VOLTAGE

\begin{tabular}{llcccc}
\hline \hline WUR & Comp. & $\begin{array}{c}\text { Power } \\
\text { consumption } \\
(\mathrm{nW})\end{array}$ & $\begin{array}{c}\text { Offset } \\
\text { voltage } \\
(\mathrm{mV})\end{array}$ & $\begin{array}{c}\text { Sensitivity } \\
(\mathrm{dBm})\end{array}$ & $\begin{array}{c}\text { Range } \\
(\mathrm{m})\end{array}$ \\
\hline 1 & TLV & 152 & 2.2 & -32 & 7 \\
2 & 3691 & 365 & 1.8 & -42 & 22 \\
3 & AS1976 & 1196 & 0.3 & -55 & 50 \\
\hline \hline
\end{tabular}

table show that the current consumption is higher for the comparator with a lower voltage offset. However, as explained in the previous section, the voltage offset affects the sensitivity of the WUR.

The proposed design (Fig. 5) uses an adaptive threshold mechanism that keeps the inverted input of the comparator at half of the input signal level. With this approach, the power consumption of the circuit is reduced since, instead of a voltage divider, the signal from the antenna is used for generating the threshold. The adaptive threshold mechanism has been designed using a simple RC circuit $\left(R_{1}-C_{5}\right)$ connected to the inverted input of the comparator while the noninverted input is directly connected to the signal from the envelope detector allowing the comparator to detect both weak and strong signals effectively.

\section{Preamble Detector}

Following the comparator, there is the preamble detector which generates the interrupt and the digital data. We used a specific preamble in the wake-up message which can be detected by this passive circuit. The preamble is an OOKmodulated signal (Fig. 6) with a specific length (number of bytes) sent at a specific bit rate (frequency $f_{\mathrm{pa}}$ ). The preamble detector is responsible for generating a wake-up interrupt for a microcontroller (or other IC) only if a preamble with the defined frequency has been received. To reject signals with the frequency lower than $f_{\mathrm{pa}}$, a low-pass filter with a cut-off frequency lower or equal to $f_{\text {pa }}$ can be implemented, as shown in Fig. $5\left(R_{2}\right.$ and $\left.C_{6}\right)$. At the rising edge of the comparator, $C_{6}$ will gradually start to charge. At the falling edge of the comparator, for signals with frequency $f<f_{\mathrm{pa}}$, capacitor $C_{6}$ will be completely discharged only through resistor $R_{3}$ by the time the next rising edge occurs on the output of the comparator. In this way, the signals with frequencies $f<f_{\text {pa }}$ will not be able to charge the capacitor $C_{6}$ to a level that can trigger an interrupt for a microcontroller $\left(0.66 \mathrm{~V}_{\mathrm{CC}}\right)$. The Schottky diode $\left(D_{5}\right)$ on the output of the comparator does not allow the capacitor $C_{6}$ to discharge through $R_{2}$ and the comparator output when the output of the comparator is grounded $(0 \mathrm{~V})$. As in Fig. 6, this mechanism is able to generate the data from the WUR which can be detected by a microcontroller or an IC.

\section{Matching Network}

Once the components are selected, the working frequency has to be defined to design needed impedance matching

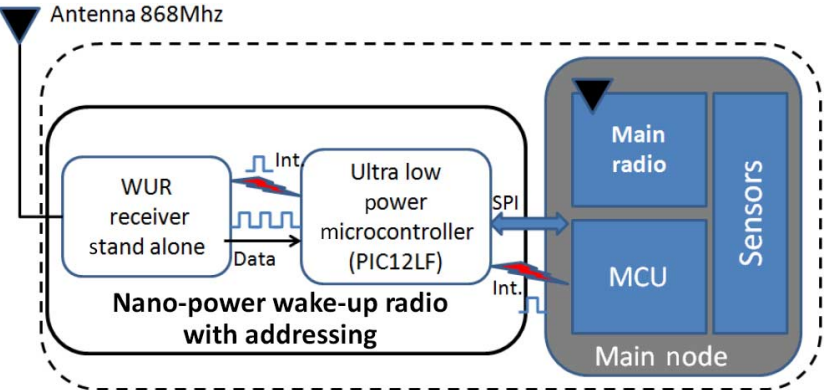

Fig. 7. Nano-power wake up radio with addressing capability provided by the PIC12LF ultra low power microcontroller.

network using advance design system (ADS). In the proposed implementation, $868 \mathrm{MHz}$ was selected which has a good tradeoff between antenna size and range, and it is commonly used in WSNs. An equivalent model was built in ADS using SPICE parameters of the HSMS-285c diode.

The matching network evaluated by ADS consists of an LC circuit, $L=74 \mathrm{nH}$ and $C=1.2 \mathrm{pF}$. This matching has to be taken into account as initial evaluation. The exact value of the $L$ and $C$ has to be measured directly on the board using the S11 parameter measurements to have optimal matching. This concept will be described in more details in the next section.

\section{E. Ultra-Low Power Addressing}

In this section, we propose a solution to add addressing and create a Nanopower WUR with addressing capability to evaluate the benefits on the overall reduction in power consumption. Fig. 7 shows the main blocks of the proposed solution. The first block is WUR presented in the previous section with sensitivity from -32 to $-55 \mathrm{dBm}$, depending on the selected comparator. The addressing block is implemented to further avoid FP messages and wake up the node only when intended. In fact, this second block generates the final wake-up signal for the main microcontroller only if the first byte received is the correct address. The main microcontroller can set its address dynamically during run time through the SPI port and also send and receive commands. In the presented architecture, this task is done by an 8-bit ultra-low power microcontroller from microchip (PIC12LF1552) which consumes only $40 \mathrm{nW}$ at $2 \mathrm{~V}$ of sleep power. So, the overall power consumption in listening mode is increased for only $40 \mathrm{nW}$ with respect to the power consumption of Table I. The PIC can be also bypassed, and the interrupt and data after the first block can be sent directly to the main microcontroller.

\section{EXPERIMENTAL SETUP}

To evaluate the functionality and to verify the performance of the proposed WUR in terms of power consumption, sensitivity, latency, and data addressing capabilities, the wake-up receiver was developed, and measurements were carried out for both versions with and without addressing capability. The prototype platform was developed using the proposed WUR connected to the PCB with CC430F5147 system-on-chip (SoC) from TI. 


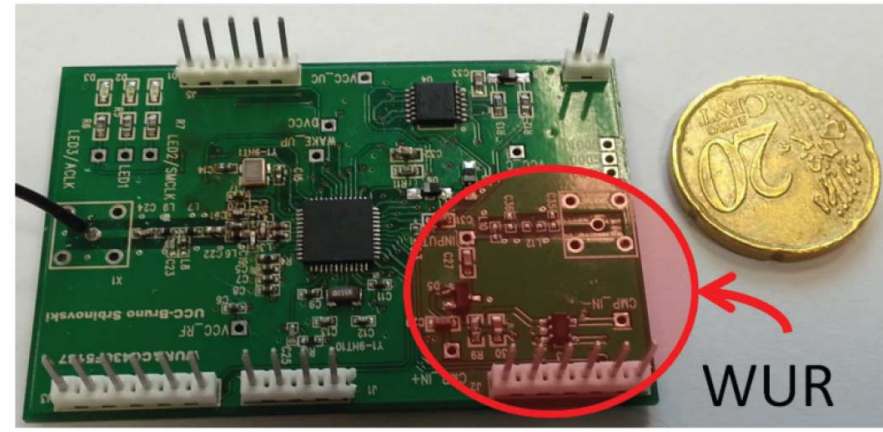

Fig. 8. First prototyped platform (WUR and CC430F5147).

The SoC is an ultra-low power microcontroller with an integrated $\mathrm{RF}$ transceiver core able to communicate at $868 \mathrm{MHz}$ with OOK modulation. The prototype platform is presented in Fig. 8. It has two separate antennas, one for the main transceiver and one for the wake-up receiver. Although the receiver and the transmitter on chip are tuned to $868 \mathrm{MHz}$, in a future devel-opment, the prototype can share a single antenna. This choice provided a more accurate measurement of the wake-up receiver which will not be affected by the antenna switching (RF mul-tiplexer). For the wake up with addressing capability (Fig. 7), we developed a new prototype including the wake-up receiver and the PIC microcontroller in a single PCB. The CC430F5147 was used as a transmitter. As we mentioned before, the trans-mitter was tuned to send an OOK modulated signal at $868 \mathrm{MHz}$ and $10 \mathrm{kbit} / \mathrm{s}$ or $f_{\mathrm{pa}}=1 \mathrm{kHz}$. A 2-bit preamble has been used beforehand to send the command/ address. The values of the resistors and capacitors (Fig. 5) used for the interferences avoid-ance circuit were as follows: $R_{2}$ $=1 \mathrm{k} \Omega ; R_{3}=100 \mathrm{k} \Omega$; and $C_{6}=200 \mathrm{nF}$. The values of the RC filter for the comparator ref-erence are $C_{3}=22 \mathrm{pF} C_{5}=1 \mathrm{nF}$. All the three configurations with the three proposed comparators have been implemented and tested, and the experimental results are presented in next section.

\section{EXPERIMENTAL Verification}

In this section, we present the experimental results and the simulations, in order to evaluate the benefits of the proposed solution compared with previous works. Moreover, we evaluate the two versions, with and without on-board addressing.

\section{A. Wake-Up Radio Features Measurements}

Minimal latency evaluation: Due to the interference avoidance filter (Fig. 5) and the value of the RC filter selected in our experimental setup, the WUR generates wake-up inter-rupts only if the preamble is detected for $60 \mu$ s that is also the latency of the wake-up signal. We also measured the minimal latency achieved by eliminating the interferences avoidance circuit which is only around $8 \mu \mathrm{s}$. It is clear that in that condition, the wake-up radio can provide a high number of FPs due to the interference, so it is convenient to keep the interference avoidance circuit and tune the RC filter according to the desirable latency.

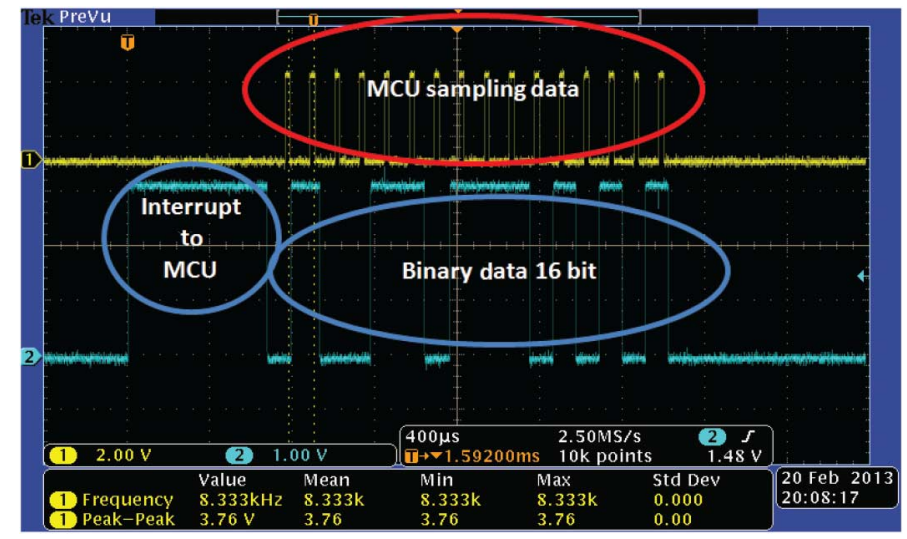

Fig. 9. Wake-up signal and data (blue) generated from the WUR, and sample period of the microcontroller to acquire the data.

Latency is also a key feature for the transmission duration and the overall consumption of the transmitting node. To evaluate this power consumption, we measured the transmission power of the CC430F5147 microcontroller. The average power consumption measured when transmitting in OOK with $+10 \mathrm{dBm}$ output power was $105 \mathrm{~mW}$. This is a considerable transmission power especially if WUR architectures need a long preamble of hundreds of milliseconds as in [9] and [24].

Address processing: To evaluate the capability to receive data and use this data for addressing or commands, firmware on the microcontroller was developed to sample the data after receiving the wake-up interrupt. Fig. 9 shows the waveform of the wake-up receiver in blue (bottom plot) and the sample time of the microcontroller in yellow (top plot). After the interrupt has been generated and the microcontroller wakes up, it starts to acquire the data until the wake-up interrupt becomes low again. In this case, a 16-bit packet was sent and correctly received by the microcontroller.

Impedance matching: A network analyzer was used to measure the S11 parameter of the previously determined matching circuit using ASD tool. Measurements of the S11 parameter showed that the matching is not optimized for $868 \mathrm{MHz}$ but for $3.1 \mathrm{GHz}$. The reason for this error is due to imperfect model of the diodes and comparator, the PCB effect, and other side effects when developing board (such as the antenna connectors and soldering). Based on these measurements, we changed the $C=7 \mathrm{pF}$ and $L=22 \mathrm{nH}$, and the matching was optimized for $868 \mathrm{MHz}$ with S11 of $-29 \mathrm{~dB}$ (Fig. 10).

Power consumption and sensitivity: As we mentioned before, the comparator used affects both the power consumption and the sensitivity. Measurements of both the parameters were carried. Table I shows: 1) the measured sensitivity of three different versions of the WUR; 2) the power consumption; and 3) range in open air at $+10-\mathrm{dBm}$ transmission power and a 3dBi antenna. The maximal range achieved with the LPV7215 comparator is $50 \mathrm{~m}$ (with $-55 \mathrm{dBm}$ sensitivity) which is an interesting value for a wide range of outdoor applications. The range of $7 \mathrm{~m}$ obtained with TLV3691-based version can be used in a body area network scenario and where power consumption is critical. To evaluate the power consumption of the WUR, we 


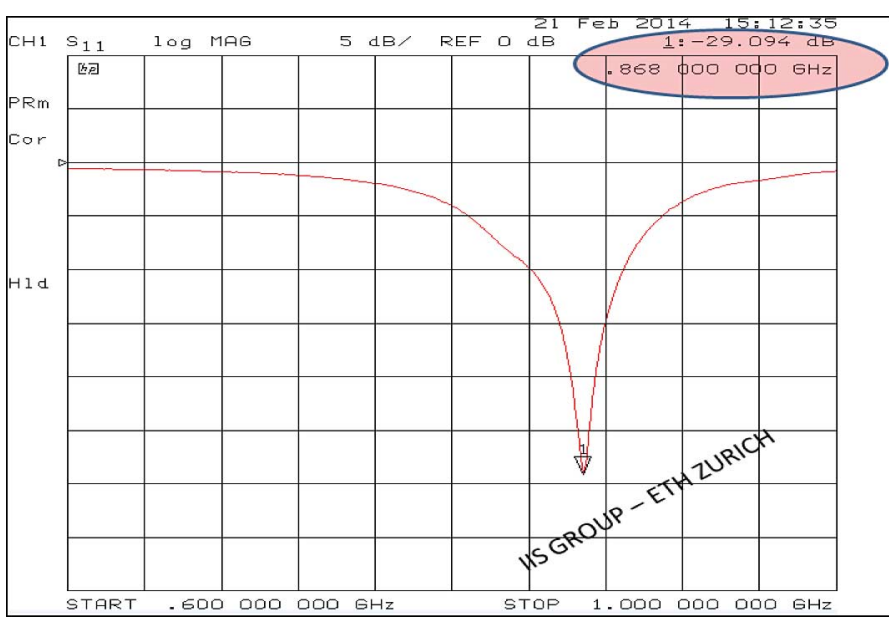

Fig. 10. S11 parameter evaluated for the impedance matching with $L$ and $C$ optimized experimentally. The new matching obtains $S 11$ $-29 \mathrm{dBm}$ at $868 \mathrm{MHz}$.

used a PCB with only the wake-up receiver soldered and supplied with $1.8 \mathrm{~V}$. We used a $100-\Omega$ shunt resistor to check the current flowing in the resistor. The static power consumption (when listening to a channel) of the presented WUR architecture is determined by the static power consumption of the comparator and can achieve only $152 \mathrm{nW}$ for $-32 \mathrm{dBm}$ and $1196 \mathrm{nW}$ for the $-55 \mathrm{dBm}$ version.

The power consumption of wake-up receiver with the addressing capability directly on board through the PIC microcontroller has been measured both in sleep mode and in active mode. The PIC microcontroller increases the overall power consumption for $40 \mathrm{nW}$ when no data are received and for $63 \mu \mathrm{W}$ during the active mode and processing the data. The power consumed by the $\mathrm{CC} 430$ in order to process the data is $600 \mu \mathrm{W}$. This confirms the benefits of using the addressing capability with the PIC over the external addressing (done by the main microcontroller), even when the microcontroller is an ultra-low power microcontroller like the CC430.

$F P$ and false negative ( $F N)$ analysis: To evaluate the benefits of the addressing and interferences avoidance circuits to reduce FPs, we tested the WUR communication in our lab. The setup has been done as follows.

1) One WUR unit with a $+3 \mathrm{dBi}$ antenna and the LPV7125 comparator has been assembled and deployed.

2) One transmitter using the $\mathrm{CC} 430 \mathrm{SoC}$ has been placed at different distances $(1,5,10,15,20,25 \mathrm{~m})$, transmitting 1000 activating messages of 8 bits representing wake-up or addressing message.

3) We sent four different addresses to wake up four different WURs.

So, only 250 messages contained the address for the tested wake-up receiver and 750 messages will contain addresses for other three WURs not evaluated.

We tested three different versions of the WUR.

V1) Without any addressing capability and interference avoidance circuits, so the WUR generates an interrupt for the main microcontroller in $8 \mu$ s whenever an OOK signal is detected.
TABLE II

WAKE-UP RADIO FP/FN PERFormance

\begin{tabular}{c|ccc}
\hline \hline \multirow{2}{*}{ Distance (m) } & V1 & FP/FN \\
& V2 & V3 \\
\hline 1 & $784 / 0$ & $752 / 0$ & $0 / 0$ \\
5 & $789 / 0$ & $756 / 0$ & $0 / 0$ \\
10 & $781 / 0$ & $753 / 0$ & $0 / 0$ \\
15 & $773 / 0$ & $753 / 0$ & $1 / 3$ \\
20 & $765 / 0$ & $752 / 0$ & $5 / 12$ \\
25 & $776 / 0$ & $756 / 0$ & $7 / 50$ \\
\hline \hline
\end{tabular}

V2) Without any addressing, but with the interference avoidance circuits, and tuned to generate an interrupt only if an OOK message long at least $60 \mu$ s is detected.

V3) With both addressing and interference avoidance circuits. So, the first bit activates the PIC microcontroller after $60 \mu \mathrm{s}$, and then the PIC processes the data and wakes up the main microcontroller only if the address matches the WUR address.

Table II shows the performance in terms of FPs and FNs for the three implementations with respect to the distance. There is a high number of FPs on both configurations without addressing. In fact, the WUR without addressing wakes up the main microcontroller also for the messages intended to wake up other nodes. Moreover, it is possible to notice the influence on the interferences in the number of FPs. On the other hand, the WUR without addressing has zero FNs. This is due to the very high probability that at least one of the transmitted 8 bits for the addresses is received and generates an interrupt. The test on the WURs with addressing improved the FP performance, especially for short ranges where the data received are more reliable due to the quality of the signal. The number of FPs and FNs increases when the range increases due to weaker signals and the corruption of the data.

\section{B. Comparison With Existing WUR Solutions and MAC Protocols}

Fig. 11 presents the three proposed WUR solutions (with and without addressing capabilities) compared to recent state of the art of the WUR solutions. The solution with the lowest power consumption (Roberts [11], $98 \mathrm{nW}$ ) has a lower sensitivity, $-41 \mathrm{dBm}$, and it does not support addressing, which makes its effective energy consumption significantly higher in real deployments (as shown in the following results). The solution with the highest $(-81 \mathrm{dBm})$ sensitivity (Milosiu [25]) also has a high power consumption $(3 \mu \mathrm{W})$ and especially high latency (484 ms). Our solution without addressing support achieves the lowest latency $(8 \mu \mathrm{s})$, but its consumption in real-world application will be increased due to excessive unnecessary wake-ups. Our solution with addressing support shows the best tradeoff in the power-latency-sensitivity space, taking into account that the WUR described by Roberts [11] does not support addressing. Moreover, as explained throughout this paper, our proposed 


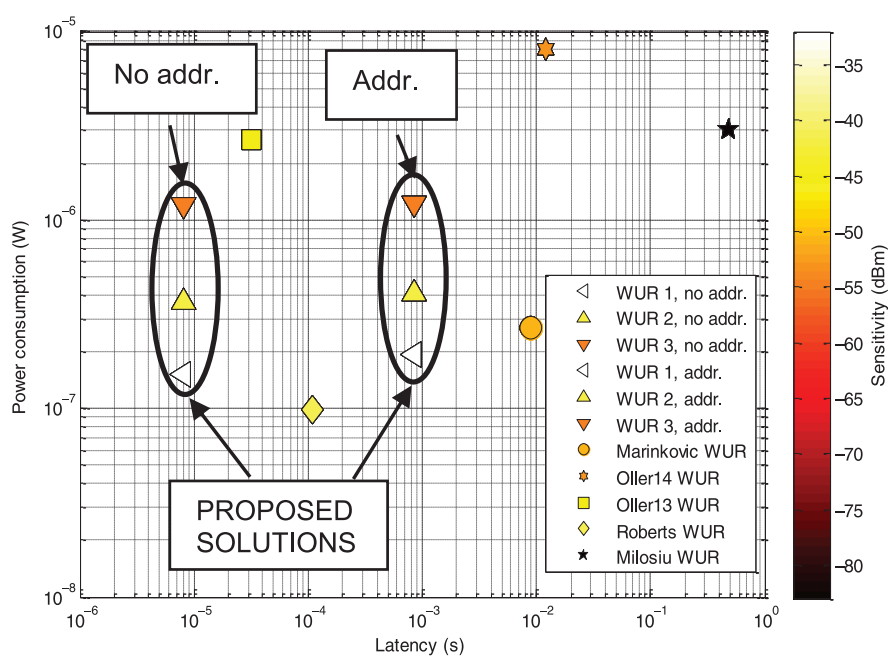

Fig. 11. Comparison of the proposed WURs (WUR 1, WUR 2, and WUR 3) with and without addressing support, with the recently published WURs, in terms of power consumption, sensitivity, and latency.

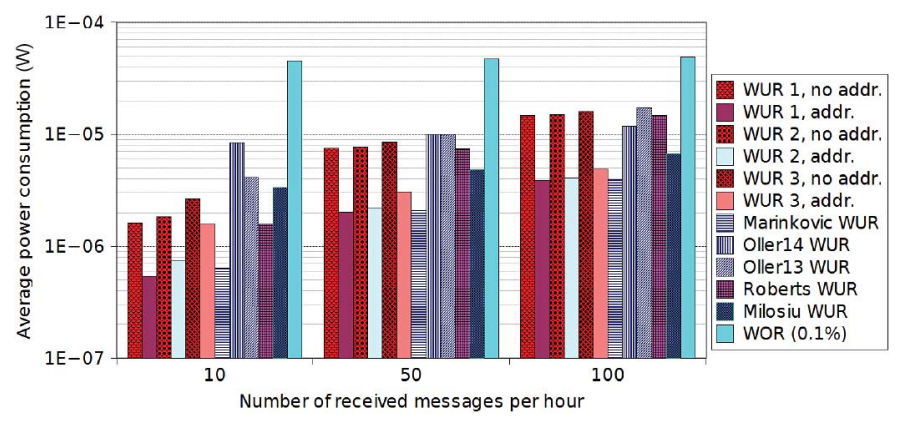

Fig. 12. Average power consumption of the communication with proposed WURs and recently published WURs, compared to the dutycycled radio (WOR) with $0.1 \%$ duty cycle, taking into account that Roberts WUR and Oller13 WUR do not have addressing possibilities.

architecture is flexible and fully adaptable for covering a wide range of frequencies and power/latency/sensitivity tradeoff.

To evaluate the benefits of using the wake-up radio over WSN protocol and for a further comparison with the state of the art, simulations using measured data were performed. Fig. 12 shows the average power consumption of different WUR solu-tions, for different numbers of received wake-up messages per hour (10, 50, and 100 messages per hour). When the wakeup message arrives, the WUR processes it (reads the address if there is any) and turns on the main transceiver to send the acknowledgment and to receive the main message. The rest of the time the system is in inactive mode. If WUR does not have addressing capabilities, each message received by the wake-up circuitry wakes up the node. We suppose the effective number of received messages for such WURs (our solutions without addressing, as well as Roberts [11] and Oller [8]) to be three times higher, due to FPs. The influence of the increased WUR receiver activity without the addressing support to the increment of their average power consumption can be seen. The average power consumption of our solutions without addressing is three to four times higher than the average power consumption of our solutions with addressing support (depending on the actual WUR and the number of messages per hour). The

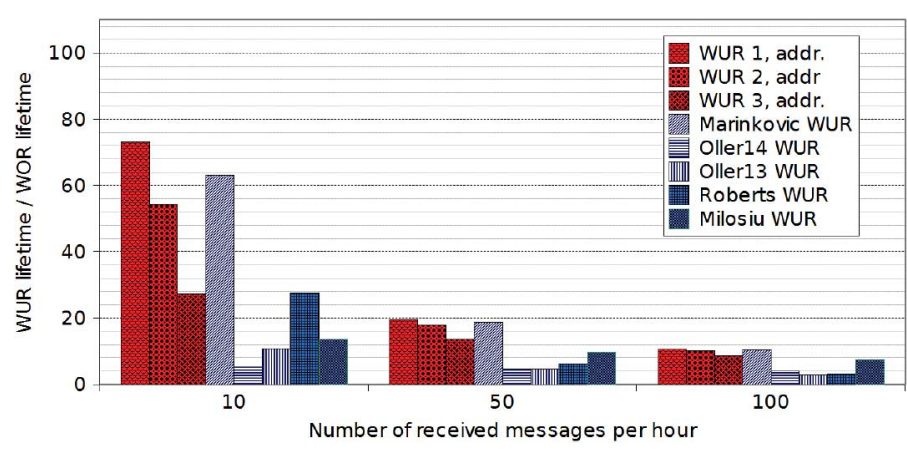

Fig. 13. Lifetime increment of the nodes with WURs against the WOR $(0.1 \%$ duty-cycle), considering the addressing possibilities. Our proposed WURs are with addressing capabilities.

number of FPs increases with the WUR range and number of transmitters, so this number can be also higher in real deployment. Thus, the increase in design complexity and in power consumption of the WUR introduced by the addressing decoding circuitry proves to be beneficial for the overall energy consumption of the WUR. WUR solutions are compared with the duty-cycling solution (WOR) with $0.1 \%$ duty cycle, where the main radio turns ON and OFF periodically regardless of the message reception. WOR is a capability featured by some TI radios that enables periodically waking up from radio sleep mode and listening for incoming packets without microcontroller interaction [26]. This feature is very common in several commercial radios to save energy. For 100 messages per hour, our WUR 3 (higher power consumption) has power levels 10 times lower, compared to the duty-cycled solution, while our least power consuming WUR consumes 12.7 times less power. For 10 messages per hour, power consumption values are much bigger (28.6 times and 84.2 times, respectively). The increase in average power consumption with more received messages is due to the fact that the major part of energy consumption is due to the activation of the main radio for receiving and transmitting the messages. For the duty-cycled solution, the average power consumption increases $7 \%$ when changed from 10 messages per hour to 100 messages per hour, due to the significant influence of radio transmission, regardless of the received messages and processing.

Finally, we evaluated a real-world scenario where a node receives a wake-up message, sends an acknowledgment, activates its sensor, acquires the data, processes the data, and sends the information in a short message to another node. Fig. 13 shows how many times each WUR solution outperforms the duty-cycled solution in terms of node's battery lifetime. As the worst case (proposed WUR 3 with addressing support, for 100 messages per hour), our solution achieves an 8 times longer lifetime.

\section{CONCLUSION}

Numerous design considerations for an ultra-low power, high-sensitivity, and fast reaction WUR for WSN have been proposed and exemplified through the development of a complete wake-up receiver subsystem with off-the-shelf components. We presented circuit topologies and optimization 
methodologies for impedance-matched passive rectifier design, nanopower interrupt generator, and addressing capability. The design techniques are supported by mathematical models, thorough simulations, and experiments on three different developments to better define the achievable tradeoffs. As many applications require addressing capabilities, the proposed approach is able to send the received data to a microcontroller or other IC to perform address detection. The proposed approach can significantly reduce power consumption of the main radio and then of the node and network. Experimental results indicate that the proposed WUR outperforms the state of the art bringing a flexible solution with ultra-low power consumption, high sensitivity, very low latency, and addressing capability. Field measurements confirmed that the proposed WUR consumes only $152 \mathrm{nW}$ in lower power version with $-32 \mathrm{dBm}$, and achieves up to $-55 \mathrm{dBm}$ of maximum sensitivity and $1.2 \mu \mathrm{W}$ power consumption. Moreover, the benefits of the addressing over no addressing are presented measuring the FP and FP rates. Finally, simulations of a real-world scenario, where implementation of different WUR solutions is compared with other wake-up solutions and duty-cycled radio protocol for WSNs, show that with our solutions, a battery lifetime prolongation of about eight times (worst-case) is achieved.

\section{References}

[1] A. Willig, "Recent and emerging topic in wireless industrial communications: A selection," IEEE Trans. Ind. Informat., vol. 4, no. 2, pp. 102-124, May 2008.

[2] I. Demirkol, C. Ersoy, and E. Onur, "Wake-up receivers for wireless sensor networks: Benefits and challenges," IEEE Wireless Commun., vol. 16, no. 4, pp. 88-96, Aug. 2009.

[3] V. Jelicic, M. Magno, D. Brunelli, V. Bilas, and L. Benini, "Analytic comparison of wake-up receivers for WSNs and benefits over the wakeon radio scheme," in Proc. 7th ACM Workshop Perform. Monit. Meas. Heterogen. Wireless Wired Netw., Oct. 2012, pp. 99-106.

[4] M. Magno, D. Boyle, D. Brunelli, E. Popovici, and L. Benini, "Ensuring survivability of resource-intensive sensor networks through ultra-low power overlays," IEEE Trans. Ind. Informat., vol. 10, no. 2, pp. 946-956, May 2014.

[5] H. Ba et al., "Passive RFID-based wake-up radios for wireless sensor networks," in Wirelessly Powered Sensor Networks and Computational RFID. New York, NY, USA: Springer, 2013, pp. 113-129.

[6] A. Castagnetti, A. Pegatoquet, T. N. Le, and M. Auguin, "A joint dutycycle and transmission power management for energy harvesting WSN," IEEE Trans. Ind. Informat., vol. 10, no. 2, pp. 928-936, May 2014.

[7] D. Spenza, M. Magno, S. Basagni, L. Benini, M. Paoli, and C. Petrioli, "Beyond duty cycling: Wake-up radio with selective awakenings for longlived wireless sensing systems," in Proc. IEEE Conf. Comput. Commun. (INFOCOM), 2015, pp. 522-530.

[8] J. Oller et al., "Design, development, and performance evaluation of a low-cost, low-power wake-up radio system for wireless sensor networks," ACM Trans. Sens. Netw., vol. 10, no. 1, p. 11, 2013.

[9] S. Marinkovic and E. Popovici, "Nano-power wireless wake up receiver with serial peripheral interface," IEEE J. Sel. Areas Commun., vol. 29, no. 8, pp. 1641-1647, Sep. 2011.

[10] B. Alírio Soares and N. Borges Carvalho, "A low-power wakeup radio for application in WSN-based indoor location systems," Int. J. Wireless Inf. Netw., vol. 20, no. 1, pp. 67-73, 2013.

[11] N. E. Roberts and D. D. Wentzloff, "A 98nW wake-up radio for wireless body area networks," in Proc. IEEE Radio Freq. Integr. Circuits Symp. (RFIC), Jun. 17-19, 2012, pp. 373-376.

[12] M. Magno and L. Benini, "An ultra low power high sensitivity wakeup radio receiver with addressing capability," in Proc. IEEE 10th Int. Conf. Wireless Mobile Comput. Netw. Commun. (WiMob), Oct. 8, 2014, pp. 92-99.

[13] K. Takahagi et al., "Low-power wake-up receiver with subthreshold CMOS circuits for wireless sensor networks," Analog Integr. Circuits Signal Process., vol. 75, no. 2, pp. 199-205, 2013.
[14] G. U. Gamm et al., "Low-power sensor node with addressable wake-up on-demand capability," Int. J. Sens. Netw., vol. 11, no. 1, pp. 48-56, 2012.

[15] S. Drago, D. M. W. Leenaerts, F. Sebastiano, L. J. Breems, K. A. A. Makinwa, and B. Nauta, "A $2.4 \mathrm{GHz} 830 \mathrm{pJ} /$ bit duty-cycled wake-up receiver with $-82 \mathrm{dBm}$ sensitivity for crystal-less wireless sensor nodes," in Proc. IEEE Int. Conf. Solid-State Circuits (ISSCC'10). San Francisco, CA, USA, Feb. 7-11, 2010, pp. 224-225.

[16] C. Hambeck, S. Mahlknecht, and T. Herndl, "A $2.4 \mu \mathrm{W}$ wake-up receiver for wireless sensor nodes with-71dBm sensitivity," in Proc. IEEE Int. Symp. Circuits Syst. (ISCAS), May 15-18, 2011, pp. 534-537.

[17] A. W. Scott and R. Frobenius, RF Measurements for Cellular Phones and Wireless Data Systems. Hoboken, NJ, USA: Wiley, 2011.

[18] P. Nintanavongsa, U. Muncuk, D. R. Lewis, and K. R. Chowdhury, "Design optimization and implementation for RF energy harvesting circuits," IEEE J. Emerg. Sel. Topics Circuits Syst., vol. 2, no. 1, pp. 24-33, Mar. 2012.

[19] R. Smat. (2012 Oct.) Introduction to Comparators, Their Parameters and Basic Applications, AN4071 Application note [Online]. Available: http:// www.st.com/st-web-ui/static/active/en/resource/technical/document/ application_note/DM00050759.pdf.

[20] Austrian Microsystems. AS1976, AS1977 Ultra Low Current, 1.8V Comparators, 1997-2007, AS1976 Datasheet [Online]. Available: http://www.ams.com/eng/Products/Power-Management/Comparators/ AS1976.

[21] Texas Instruments. (2013 Sep.) MSP430 TM SoC With RF Core, CC430F5137 Datasheet [Online]. Available: http://www.ti.com/lit/ds/ symlink/cc430f5137.pdf.

[22] Avago Technologies US. (2009 May 29). HSMS285x-Series Datasheet [Online]. Available: http://www.avagotech.com/docs/AV02-1377EN.

[23] V. Jelicic, M. Magno, D. Brunelli, V. Bilas, and L. Benini, "Benefits of wake-up radio in energy-efficient multimodal surveillance wireless sensor network," IEEE Sensors J., vol. 14, no. 9, pp. 3210-3220, Sep. 2014.

[24] J. Oller, I. Demirkol, J. Casademont, J. Paradells, G. Gamm, and L. Reindl, "Performance evaluation and comparative analysis of subcarrier modulation wake-up radio systems for energy-efficient wireless sensor networks," Sensors, vol. 14, pp. 22-51, 2014.

[25] H. Milosiu et al., "A 3- $\mu$ W 868-MHz wake-up receiver with - $83 \mathrm{dBm}$ sensitivity and scalable data rate," in Proc. ESSCIRC, Bucharest, Romania, Sep. 16-20, 2013, pp. 387-390.

[26] D. Dang, "CC430 wake-on-radio functionality," Texas Instrum., Dallas, TX, USA, Appl. Rep. SLAA459A, 2012.

Michele Magno (M'13-SM'16) received the M.S. and Ph.D. degrees in electronic engineer-ing from the University of Bologna, Bologna, Italy, in 2004 and 2010, respectively.

Currently, he is a Postdoctoral Researcher with Swiss Federal Institute of Technology (ETH) Zurich, Zurich, Switzerland, and a Research Fellow with the University of Bologna. He has collaborated with several universities and research centers, such as the University College Cork and the Tyndall Institute, Cork, Ireland; Imperial College London, London, U.K.; the University of Trento, Trento, Italy; Politecnico di Torino, Torino, Italy; the University of British Columbia, Vancouver, BC, Canada; etc. He has authored more than 60 papers in international journals and conferences. His research interests include wireless sensor networks, power management techniques, and extension of lifetime of batteries-operating devices and embedded video surveillance.

Vana Jelicic (S'09) received the Ph.D. and M.S. degrees in electrical engineering from the Faculty of Electrical Engineering and Computing, University of Zagreb, Zagreb, Croatia, in 2014 and 2009 , respectively.

She was a Visiting Researcher at the Department of Electrical Engineering and Computer Science, University of Bologna, Bologna, Italy, from 2010 to 2012. In 2015, she was a Postdoctoral Researcher with the Faculty of Electrical Engineering and Computing, University of Zagreb. Her research interests include smart sensors and wireless sensor networks (WSNs), with focus on power management in WSNs with high-consuming sensors. 
Bruno Srbinovski $\left(S^{\prime} 14\right)$ received the M.S. degree in electrical engineering and information technologies from the Faculty of Electrical Engineering and Information Technologies, Skopje, Macedonia, in 2011, and the M.S. degree in electronic engineering from University College Cork, Cork, Ireland, in 2013. $\mathrm{He}$ is currently working toward the Ph.D. degree at the University College Cork. His research interests include the area of wind energy harvesting, wireless sensor networks, power management, sensor interface, and digital signal.

Vedran Bilas (M'98-SM'10) received the Ph.D. degree in electrical engineering from the University of Zagreb, Zagreb, Croatia, in 1999.

$\mathrm{He}$ is a Professor heading the Laboratory for Intelligent Sensor Systems with the Faculty of Electrical Engineering and Computing, University of Zagreb. He has over 20 years of research, development, and technology transfer experience in the area of sensors and electronic systems. His research interests include the field of energy efficient intelligent and networked sensors in various application domains.

Emanuel Popovici (S'00-M'03-M'08) received the Dipl. Ing. degree in computer engi-neering from Politehnica University Timisoara, Timisoara, Romania, in 1997, and the Ph.D. degree in microelectronic engineering from the National University of Ireland, Cork, Ireland, in 2002.

$\mathrm{He}$ has been a Senior Lecturer with the Department of Electrical and Electronic Engineering, National University of Ireland, since 2002. His research interests include embedded system design for low power, reliable, and secure computing and com-munications with applications in smart LED lighting systems, biomedical, agricultural, structural health, energy control and optimization, learning, and entertainment domains. He has published widely in these areas, with more than 10 co-authored papers being distinguished at national and international levels.

Dr. Popovici co-supervised three interdisciplinary teams who achieved top awards from the IEEE/International Business Machines (IBM) Smarter Planet Challenge in 2011, 2013, and 2014.

Luca Benini (S'94-M'97-SM'04-F'07) received the Ph.D. degree in electrical engineering from Stanford University, Stanford, CA, USA, in 1997.

$\mathrm{He}$ is a Full Professor with the University of Bologna, Bologna, Italy, and he is the Chair of Digital Circuits and Systems with Swiss Federal Institute of Technology (ETHZ), Zurich, Switzerland. $\mathrm{He}$ served as Chief Architect for the Platform2012/STHORM project in STmicroelectronics, Grenoble, France, in the period 2009-2013. He has held Visiting and Consulting Researcher positions at École polytechnique fédérale de Lausanne (EPFL), Lausanne, Switzerland; IMEC, Leuven, Belgium; Hewlett-Packard Laboratories; and Stanford University, Stanford, CA, USA. He has authored more than 700 papers in peer-reviewed international journals and conferences, four books, and several book chapters. His research interests include energy-efficient system design, multicore state of charge design, energy-efficient smart sensors, and sensor networks for biomedical and ambient intelligence applications.

Dr. Benini is a Member of Academia Europaea. 